\title{
Facile Synthesis of Hollow Spheres of Sulfonated Polyanilines
}

\author{
Kakarla Raghava RedDy, ${ }^{1}$ Kwang-Pill LEE, ${ }^{1,2, \dagger}$ \\ Anantha Iyengar GopAlAn, ${ }^{1,2,3}$ and Ali Md SHOWKAT ${ }^{1}$ \\ ${ }^{1}$ Advanced Nanomaterials Laboratory, Department of Chemistry, Kyungpook National University, \\ Daegu 702-701, South Korea \\ ${ }^{2}$ Nano Practical Application Center, Deagu 704-230, South Korea \\ ${ }^{3}$ Department of Industrial Chemistry, Alagappa University, Karaikudi-630 003, Tamil Nadu, India
}

(Received September 26, 2005; Accepted November 21, 2005; Published April 15, 2006)

\begin{abstract}
Core-shell type sulfonated polyaniline (SPAN)-iron oxide nanoparticles (IONP) composites (IONPSPAN-NC) were prepared. The interactions between the positve and negative charges on the surface of IONP and sulfonic acid and amine groups, respectively, in SPAN form the basis for core-shell structure for the composite. Sulfonated polyaniline hollow spheres (SPAN-HS) were generated by preferential dissolution of IONP from the composite. TEM photograph of IONP-SPAN-NC reveals that IONPs are 'glued' to SPAN chains and hollow spheres are formed on removal of IONP. Morphology, optical and magnetic properties of the IONP-SPAN-NC and SPAN-HS were compared. [DOI 10.1295/polymj.38.349]
\end{abstract}

KEY WORDS Sulfonated Polyaniline / Iron Oxide / Nanoparticles / Composite / Hollow Sphere /

Polyaniline (PANI) and its derivatives are considered as potential materials for optical, micro and nanoelectronic applications such as light emitting diodes, photovoltaic devices, chemical sensors, actuators, drug delivery and energy storage devices, ${ }^{1-5}$ due to their advantageous properties. The oxidation state and extent of doping are the key factors that determine the electrochemical properties of polyaniline class of materials. Doping can be achieved externally with acids like $\mathrm{HCl}$ or $\mathrm{HClO}_{4}$ or internally (self-doping). In sulfonated polyaniline (SPAN), self-doping occurs through interaction between $-\mathrm{NH}$ group of PANI and sulfonic acid group present in the phenyl rings. SPAN has stable and reproducible electrical properties, $\mathrm{pH}$ independent electrical conductivity, processing ability and higher thermal stability than PANI. ${ }^{6,7}$

Applications of conducting polymers in nanodevices require the use of one dimensional (1D) nanostructures and thus synthesis of nanoscale conducting polymers (nanotubes/-rods/-wires/-fibers) has attracted considerable attention. ${ }^{8}$ 1-D PANI nanostructures have been generated by chemical and electrochemical methods through polymerization of the monomer with the aid of either a "hard' or a "soft" template. Examples of hard templates include zeolite channels, track-etched polycarbonate, and anodized alumina. ${ }^{9,10}$ Soft templates, such as surfactants, micelles, liquid crystals, thiolated cyclodextrins and polyacids, ${ }^{11,12}$ have been reported to be capable of directing the growth of polyaniline 1-D nanostructures with diameters smaller than $100 \mathrm{~nm}$. Physical methods, ${ }^{13,14}$ in- cluding electrospinning and mechanical stretching have also been used to make polyaniline nanofibers. Studies have been reported on blending of PANI with inorganic nanoparticles to result PANI based hybrid nanostructures. ${ }^{15,16}$ Formation of conducting polymer nanotube inside the channels of MCM-41 (mesoporous silica) has been recently reported. ${ }^{17,18}$

Capsules and hollow spheres have wider applications in catalysis, delivery and controlled release, opto-electronics, microcavity resonance and photonic crystals. ${ }^{19}$ Colloidal particles have been used as templates for the preparation of hollow nanospheres. ${ }^{20-22}$ Layer-by-layer assembly of polymeric or inorganic/ metallic materials over template particles has been utilized to form an initial composite and that acts as a precursor for the preparation of hollow spheres. ${ }^{23-25}$ To our knowledge, no systematic procedure has been established for the preparation of nanostructures of functionalized PANI like SPAN.

In this paper, we are reporting a simple and facile approach for the preparation of sulfonated polyaniline hollowspheres (SPAN-HS). Preparation of SPAN-HS involved three steps (Scheme 1): (i) preparation of surface charged $\mathrm{Fe}_{3} \mathrm{O}_{4}$ nanoparticles (IONP) as core, (ii) preparation of $\mathrm{Fe}_{3} \mathrm{O}_{4}$-SPAN core-shell type nanocomposite (IONP-SPAN-NC), (2,5-diaminobenene sulfonic acid (DABSA) and aniline were used to form SPAN coating over IONP). The presence of two amine groups in DABSA can result cross-linking during polymerization. And, (iii) preferential removal of IONP $^{26}$ from IONP-SPAN-NC to get SPAN-HS.

${ }^{\dagger}$ To whom correspondence should be addressed (Tel: +82-539505901, Fax: +82-539528104, E-mail: kplee@knu.ac.kr). 


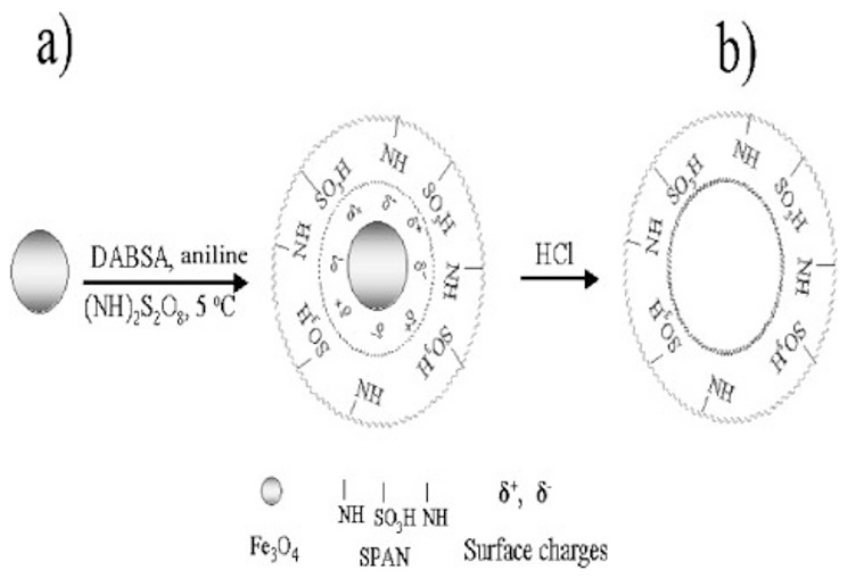

Scheme 1. (a) Preparation of IONP-SPAN-NC and (b) SPANHS.

\section{EXPERIMENTAL}

\section{Chemicals}

Aniline (reagent grade, Oriental) was distilled under reduced pressure and stored below $0{ }^{\circ} \mathrm{C}$. Other reagents, 2,5-diaminobenzenesulfonic acid (DABSA), iron (II) chloride tetra hydrate, iron (III) chloride hexahydrate, ammonium peroxydisulfate, (APS), sodium hydroxide, hydrochloric acid and ethanol were of analytical grade samples and used without further purification. Milli-Q purified water was used for all experiments.

\section{Preparation of Surface Bound $\mathrm{Fe}_{3} \mathrm{O}_{4}$ Nanoparticles (IONP)}

$5.2 \mathrm{~g}(0.77 \mathrm{M})$ of $\mathrm{FeCl}_{3} \cdot 6 \mathrm{H}_{2} \mathrm{O}$ and $2.0 \mathrm{~g}(0.402 \mathrm{M})$ of $\mathrm{FeCl}_{2} \cdot 4 \mathrm{H}_{2} \mathrm{O}$ were dissolved in $25 \mathrm{~mL}$ of $0.40 \mathrm{M}$ $\mathrm{HCl}$. The solution of mixed iron-salts was added drop-wise into $250 \mathrm{~mL}$ of $1.5 \mathrm{M} \mathrm{NaOH}$ solution under vigorous stirring for $30 \mathrm{~min}$ in the $\mathrm{N}_{2}$ atmosphere. The resulting solution was centrifuged at $4000 \mathrm{rpm}$, washed with deionised water and dried in vacuum at $70^{\circ} \mathrm{C}$ for $6 \mathrm{~h}$. IONP was collected as brown black residue.

\section{Preparation of IONP-SPAN-NC}

$0.2 \mathrm{~g}$ of IONP was dispersed in a solution of $0.02 \mathrm{M}$ of DABSA and $0.2 \mathrm{M}$ of aniline in $80 \mathrm{~mL}$ of ethanol/ water ( $4: 1$ by volume) and the solution was sonicated for $20 \mathrm{~min}$. Polymerization was carried out at $5^{\circ} \mathrm{C}$ by the drop wise addition of $20 \mathrm{~mL}$ of $1 \mathrm{M}$ APS in $4: 1 \mathrm{v} / \mathrm{v}$ ethanol/water with simultaneous vigorous stirring. A green colour precipitate (IONP-SPAN-NC) was obtained. The green precipitate was washed three times with ethanol/water (4:1) and dried at vacuum.

\section{Preparation of SPAN-HS}

IONP-SPAN-NC was treated with $3 \mathrm{~N} \mathrm{HCl}$ for $24 \mathrm{~h}$, filtered, washed with deionised water and dried in vacuum for $6 \mathrm{~h}$.

\section{Characterization}

Fourier transform infrared (FT-IR) spectra were recorded on a Bruker IFS 66v FT-IR in the region of 400 to $4000 \mathrm{~cm}^{-1}$ using $\mathrm{KBr}$ pellets. Ultraviolet-visible spectra were recorded in $N, N$-dimethylformamide (DMF) solution by using Beckman UV-vis (DU7500) spectrophotometer. Morphology of SPAN-NC and SPAN-HS was determined by using transmission electron microscope (TEM) (EF-TEM, EM 912 Omega, Carl Zeiss, Germany) with an accelerating voltage of $100 \mathrm{kV}$ and high voltage transmission electron microscopy (HVTEM), respectively. Thermogravimetric analysis of IONP-SPAN-NC and SPAN-HS was made using a Dupont 9900/2100 TGA at a heating rate of $10^{\circ} \mathrm{C} / \mathrm{min}$ under a nitrogen atmosphere over a temperature range of $30-650{ }^{\circ} \mathrm{C}$. X-ray diffraction (XRD) patterns of SPAN-NC and SPAN-HS were recorded using Rigaku Diffractomer with Nickel filtered $\mathrm{CuK} \alpha$ radiation.

\section{RESULTS AND DISCUSSIONS}

For inducing the formation of core-shell nanocomposite IONP and SPAN through molecular interactions, IONPs with excess surface charges were prepared using mixture of $\mathrm{Fe}(\mathrm{II})$ and $\mathrm{Fe}(\mathrm{III})$ ions. The combined use of $\mathrm{Fe}$ (II) and Fe(III) salts would facilitated the packing of iron cations in spinel structure of $\mathrm{Fe}_{3} \mathrm{O}_{4}{ }^{27}$ and to have excess negative charge (through oxide ions) on the surface of nanoparticles. ${ }^{28,29}$ It is known from the literature that formation of $\mathrm{Fe}_{3} \mathrm{O}_{4}$ from aqueous solutions of iron salts involves complex multiple steps including deprotonation, hydrolysis, oxidation, precipitation, nucleation, crystallization, dehydroxylation, and/or dehydration. ${ }^{30}$ Precipitation of pure $\mathrm{Fe}^{3+}$ ions typically produces amorphous hydrated oxyhydroxide that subsequently converts into $\mathrm{Fe}_{3} \mathrm{O}_{4} \cdot \mathrm{Fe}_{3} \mathrm{O}_{4}$ particles thus have corundum structure with the oxide ions forming a hexagonally close packed array having $\mathrm{Fe}^{3+}$ ions in the octahedral interstices. The preference of $\mathrm{Fe}^{2+}$ ions over $\mathrm{Fe}^{3+}$ ions in the octahedral interstices promotes the formation of $\mathrm{Fe}_{3} \mathrm{O}_{4}$ with cation-deficient spinel structure of the $\mathrm{A}\left[\mathrm{B}_{2}\right] \mathrm{O}_{4}$ type. $\mathrm{Fe}^{3+}$ ions occupy tetrahedral interstices in this structure. ${ }^{31}$ Thus, for the synthesis of the $\mathrm{Fe}_{3} \mathrm{O}_{4}$ nanoparticles, we used a mixture of $\mathrm{FeCl}_{3}$ and $\mathrm{FeCl}_{2}$ with an $\mathrm{Fe}^{3+} / \mathrm{Fe}^{2+}$ mole ratio of 2 to facilitate the packing of the iron cations in the spinel structure of $\mathrm{Fe}_{3} \mathrm{O}_{4} \cdot{ }^{27}$

Oxidative polymerization was performed for a solution consisting of aniline, DABSA and IONP to obtain IONP-SPAN-NC. The monomers (aniline and DABSA) were adsorbed on the surface of IONP and polymerized on the surface of IONP. Copolymerization of aniline and DABSA resulted sulfonated poly- 
aniline (SPAN). DABSA played two important roles in the formation of SPAN. It acts as a co-monomer with aniline to provide sulfonic acid containing aniline units in the resultant polymer as well as a crosslinker during the polymerization. It is to be noted that DABSA has two $-\mathrm{NH}_{2}$ groups. A highly cross-linked SPAN structure was expected due to the involvement of both $-\mathrm{NH}_{2}$ groups in DABSA groups for the formation of SPAN. Additionally, electrostatic attractions of the positive and negative charges on the surface of spherical IONP with sulfonic acid groups and protanated imine groups in SPAN could keep SPAN over the surface of IONP. Hence, SPAN with highly crosslinked rigid structure was expected to present was on the surface of IONP to result IONP (core)-SPAN (shell) composites.

A molar ratio of 1:10 between aniline and DABSA resulted the core-shell IONP-SPAN-NC. However, when the ratio aniline to DABSA was changed with increasing proportion of aniline or DABSA, the resultant precipitate, IONP-SPAN-NC, did not posses a core-shell type morphology. Presence of sulfonic acid groups in DABSA bound the SPAN with IONP through electrostatic interactions. It is important to note that the core-shell type composite was not obtained when the polymerization medium contained aniline alone. This adds support to the role of sulfonic acid groups in DABSA in forming the core-shell type composite.

Liqiang $\mathrm{Xu}$ et al. ${ }^{26}$ encapsulated iron oxide nanowires in carbon microtubes and carbon microtubes and further subjected with aqueous $\mathrm{HCl}$ to remove the iron oxide nanowires from the encapsulated structure. A similar methodology was adopted by us for the preparation of SPAN-HS. Upon treatment of IONPSPAN-NC with aqueous $3 \mathrm{~N} \mathrm{HCl}$, IONP (core) was preferentially removed from the composite to form SPAN (shell) matrix alone. Due to the removal of spherical shaped IONP, the morphology of the SPAN was expected to be hollow sphere.

The high voltage transmission electron microscopy (HVTEM) micrograph clearly reveals the hollow sphere nature after the removal of IONPs from IONP-SPAN-NC (Figure 1). A thin shell of SPAN with a fairly larger inner cavity is evident from the HVTEM micrograph (Figure 1). The hollow spheres have smooth outer and inner surfaces. The thickness of SPAN-HS seems to be uniform. The combination of DABSA with aniline provides effective cross linking during polymerization and causes a rigid spherical structure to SPAN-HS.

The average diameter of the IONP nanoparticles (D, $\AA$ A) was estimated by using the Scherrer's equation. ${ }^{32}$

$$
\mathrm{D}=\mathrm{K} \lambda / \beta \cos \theta
$$
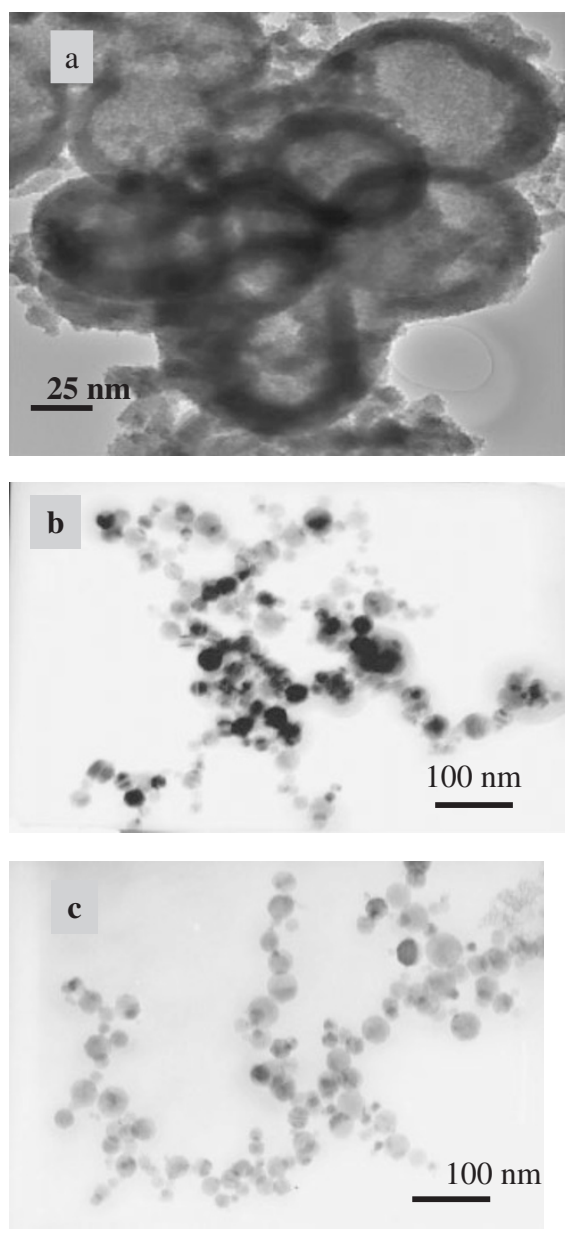

Figure 1. HVTEM images of (a) SPAN-HS and TEM images of (b) IONP-SPAN-NC and (c) SPAN-HS.

where $\lambda$ is the $\mathrm{X}$-ray wavelength $(1.5406 \AA), \mathrm{K}$ is the shape factor, $\mathrm{D}$ is average diameter of the crystals (in angstroms), $\theta$ is the Bragg angle (in degrees), $\beta$ is the full width at half-maximum (fwhm) expressed in units of $2 \theta$. The value of ' $K$ ' depends on several factors, including the Miller index of the reflecting plane and the shape of the crystals. ${ }^{33,34}$ If the shape is unknown, $\mathrm{K}$ is often assumed as 0.89 . The reflecting peak at $2 \theta=35.7^{\circ}(\mathrm{d}=2.508 \AA)$ was chosen and the average size of the IONP nanoparticles was determined to be $4.6 \mathrm{~nm}$. This is in good agreement with TEM image of IONP-SPAN-NC. TEM micrographs (Figure 1) clearly reveal that the particles of IONP-SPAN-NCs are nearly spherical with a diameter range of 15-25 $\mathrm{nm}$. In the composite, IONP (dark shaded) is glued by SPAN (light shaded). The inner diameter of the hollow spheres varies and this variation is in accordance with the diameter of IONPs. However, we could not estimate the actual size of hollow sphere probably due to poor contrast between the empty space and shell.

We have obtained evidences from UV-vis and FT-IR spectroscopy for the removal of IONP from IONP-SPAN-NC in the process of making SPAN- 


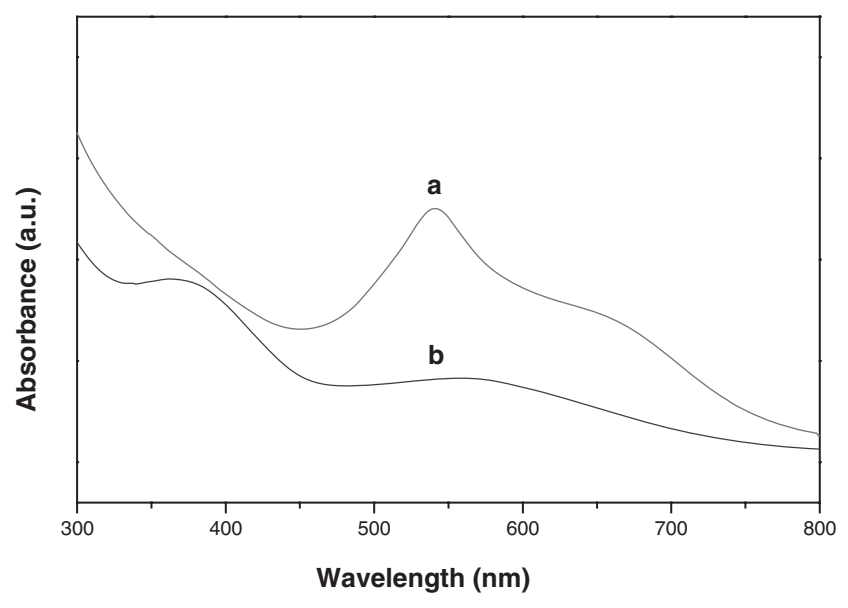

Figure 2. UV-vis spectra of (a) IONP-SPAN-NC and (b) SPAN-HS.

HS. A comparison of UV-vis spectra (Figure 2) of IONP-SPAN-NC and SPAN-HS indicates the differences in the electronic states of SPAN between them. The spectrum of SPAN-HS (Figure 2a) shows a band around $360 \mathrm{~nm}$ for $\pi \rightarrow \pi^{*}$ transition of benzenoid units and a band around $570 \mathrm{~nm}$ for the exitonic transition of quinoid diimine units arising from self-doping from $-\mathrm{SO}_{3} \mathrm{H}^{35,36}$

The ratio of absorbance's of benzenoid to quinoid bands can be taken as a measure of oxidation state in polyaniline type conducting polymer. ${ }^{37}$ Generally, emeraldine structure of PANI will have nearly equal number of benzenoid and quinoid units. Hence, the ratio of absorbance of benzenoid to quinoid bands will be unity, if PANI possesses emeraldine structure. The ratio of absorbance's of benzenoid to quinoid bands, a measure of doping of PANI, is higher than unity for SPAN-HS indicating the presence of more of benzenoid units in SPAN-HS. It is pertinent to note that UV-vis spectrum of IONP-SPAN-NC composite has a very weak band around $370 \mathrm{~nm}$. This indicates that benzenoid units of SPAN interact with surface charges of IONP and transform into quinoid units in IONPSPAN-NC. The exitonic band for IONP-SPAN-NC appears around $540 \mathrm{~nm}$. An additional band can be seen around $660 \mathrm{~nm}$. The origin of the band around $660 \mathrm{~nm}$ is not known. Removal of IONP from IONP-SPAN-NC caused increase in the absorbance ratio of benzenoid to quinoid units. When IONP was removed from IONP-SPAN-NC, the interactions of $-\mathrm{SO}_{3}{ }^{-}$groups with IONP became absent. As a consequence, a portion of quinoid units might be converted to benzenoid units. Due to that more benzenoid structures are expected in SPAN-HS than in IONP-SPANNC. This is evident from the pronounced peak around $370 \mathrm{~nm}$ for SPAN-HC. The shifts in exitonic band and variations in the proportion of quinoid diimine units between IONP-SPAN-NC and SPAN-HS support the

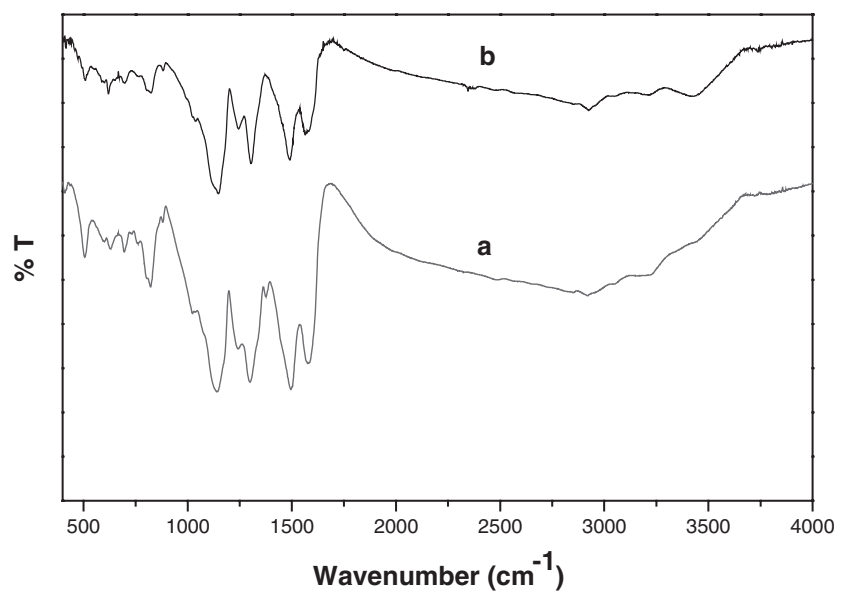

Figure 3. FT-IR spectra of (a) IONP-SPAN-NC and (b) SPAN-HS.

removal of IONP from the IONP-SPAN-NC. Presence of $-\mathrm{SO}_{3} \mathrm{H}$ groups in IONC-SPAN-NC, make the nanocomposite to soluble in organic solvents such as chloroform, dichloromethane, toluene. But, PANI$\mathrm{Fe}_{3} \mathrm{O}_{4}$ composite was not soluble in organic solvents ${ }^{35}$ due to absence of $-\mathrm{SO}_{3} \mathrm{H}$ groups. Additionally, SPAN-HS did not show super paramagnetic behavior that has been noticed for magnetic nanocomposites like IONP-SPAN-NC. ${ }^{38}$

A comparison of FT-IR spectra of SPAN-HS and IONP-SPAN-NC (Figure 3) informs that SPAN-HS and IONP-SPAN-NC have different proportions of benzeniod and quinoid units. The peaks representing the $\mathrm{C}-\mathrm{C}$ stretching of the benzenoid ring, quinoid ring and $\mathrm{C}-\mathrm{H}$ bending vibrations of quinoid/benzenoid ring appeared at 1564,1490 and $1148 \mathrm{~cm}^{-1}$, respectively for SPAN-HS. However, for IONP-SPAN-NC, the respective peaks appeared at 1585, 1502 and $1136 \mathrm{~cm}^{-1}$, respectively.

The shifts in peak positions and ratio of intensities of benzenoid and quinoid units between SPAN-HS and IONP-SPAN-NC inform that IONP is removed from IONP-SPAN-NC while making SPAN-HS. XRD (Figure 4) and thermal analysis (Figure 5) of IONPSPAN-NC and SPAN-HS also revealed that IONP was virtually absent in SPAN-HS. The crystalline peaks at $35.5^{\circ}$ and $63.0^{\circ}$ representing the pristine IONP $^{39-41}$ are virtually absent in SPAN-HS (Figure 4), while those two peaks can also be seen in IONPSPAN-NC. Thermogram of SPAN-HS starts informs that degradation of backbone units of SPAN starts at a lower temperature $\left(280^{\circ} \mathrm{C}\right)$ in comparison to $310^{\circ} \mathrm{C}$ for IONP-SPAN-NC.

\section{CONCLUSIONS}

We demonstrated that surface charged IONP can be used as template to form SPAN-HS. We anticipate 


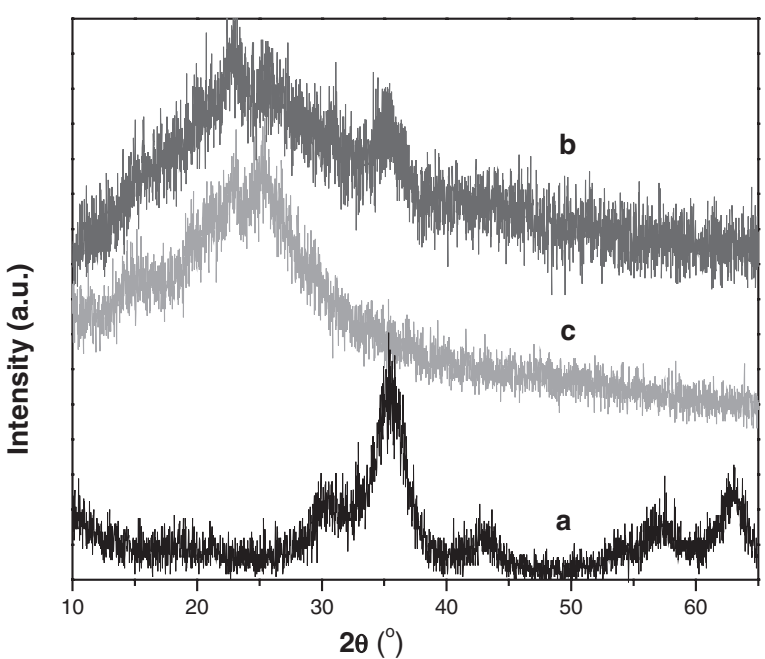

Figure 4. X-Ray scattering patterns of (a) IONP, (b) IONPSPAN-NC and (c) SPAN-HS.

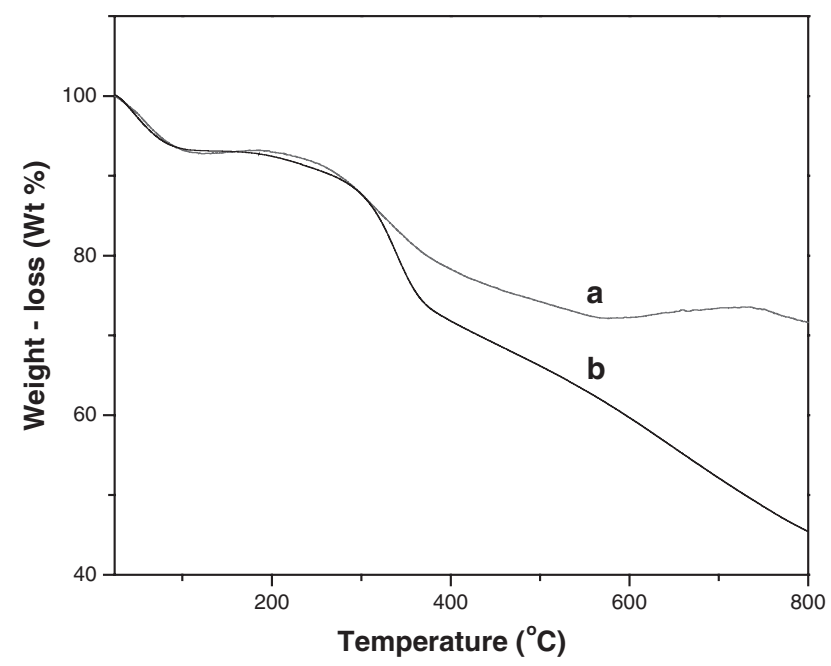

Figure 5. Thermograms of (a) IONP-SPAN-NC and (b) SPAN-HS.

that such SPAN-HS can find applications in controlled release, opto-electronics and photonic crystals.

Acknowledgment. The financial support from Korean Research Foundation Grant (KRF-2004-00500009) is greatly acknowledged. The authors acknowledge the help of centre for high volt transmission microscope, Korea Basic Science Institute, Daejon, Korea for recording the FETEM and HVTEM photographs.

\section{REFERENCES}

1. J. C. Chiang and A. G. MacDiarmid, Synth. Met., 13, 193 (1986).

2. A. G. MacDiarmid, Angew. Chem., Int. Ed., 40, 2581 (2001).

3. J. M. Yeh, S. J. Liou, C. Y. Lai, P. C. Wu, and T. Y. Tsai, Chem. Mater., 13, 1131 (2001).
4. D. Chaudhuri, A. Kumar, I. Rudra, and D. D. Sarma, $A d v$. Mater., 13, 1548 (2001).

5. W. Lu, E. Smela, P. Adams, G. Zuccarello, and B. R. Mattes, Chem. Mater., 16, 1615 (2004).

6. Y. Jiang and A. J. Epstein, J. Am. Chem. Soc., 112, 2800 (1990).

7. X. L. Wei, M. Fahlman, and A. J. Epstein, Macromolecules, 32, 3114 (1999).

8. X. Zhang, J. Zhang, Z. Liu, and C. Robinson, Chem. Commun., 16, 1852 (2004).

9. C. R. Martin, Chem. Mater., 8, 1739 (1996).

10. C. W. Wang, Z. Wang, M. K. Li, and H. L. Li, Chem. Phys. Lett., 341, 431 (2001).

11. H. J. Qiu and M. X. Wan, J. Polym. Sci., Part A: Polym. Chem., 39, 3485 (2001).

12. S. J. Choi and S. M. Park, Adv. Mater., 12, 1547 (2000).

13. D. H. Reneker and I. Chun, Nanotechnology, 7, 216 (1996).

14. H. X. He, C. Z. Li, and N. J. Tao, Appl. Phys. Lett., 78, 811 (2001).

15. M. D. Butterworth, S. A. Bell, S. P. Armes, and A. W. Simpson, J. Colloid Interface Sci., 183, 91 (1996).

16. H. X. Guo and X. P. Zhao, Opt. Mater, 22, 39 (2003).

17. K. P. Lee, A. M. Showkat, A. Gopalan, S. H. Kim, and S. H. Choi, Macromolecules, 38, 364 (2005).

18. A. M. Showkat, K. P. Lee, A. Gopalan, K. S. Kim, S. H. Choi, and H. D. Kong, Polymer, 46, 1804 (2005).

19. F. Caruso, Adv. Mater., 13, 11 (2001).

20. A. Imhof, Langmuir, 17, 3579 (2001).

21. X. C. Guo and P. Dong, Langmuir, 15, 5535 (1999).

22. M. J. Percy, C. Barthet, J. C. Lobb, M. A. Khan, S. F. Lascelles, M. Vamvakaki, and S. P. Armes, Langmuir, 16, 6913 (2000).

23. I. S. Pastoriza, B. Scholer, and F. Caruso, Adv. Funct. Mater., 11, 122 (2001).

24. F. Caruso, M. Spasova, A. Susha, M. Giersig, and R. A. Caruso, Chem. Mater., 13, 109 (2001).

25. G. Kumaraswamy, A. M. Dibaj, and F. Caruso, Langmuir, 18, 4150 (2002).

26. L. Xu, W. Zhang, Y. Ding, Y. Peng, S. Zhang, W. Yu, and Y. Qian, J. Phys. Chem. B., 108, 10859 (2004).

27. Y. S. Kang, S. Risbud, J. F. Rabolt, and P. Stroev, Chem. Mater., 8, 2209 (1996).

28. S. Morup, F. Modker, P. V. Hendriksen, and S. Linderoth, Phys. Rev. B, 52, 287 (1995).

29. L. Zhang, G. C. Papaefthymiou, and J. Y. Ying, J. Appl. Phys., 81, 6992 (1997).

30. J. L. Jambor and J. E. Dutrizac, Chem. Rev., 98, 2549 (1998).

31. "Nanophase Materials," E. Tronc, J. P. Jolivert, G. C. Hadjipanayis, and P. W. Siegel, Ed., Netherlands, 1994, p 21.

32. H. P. Klong and L. E. Alexander, "X-Ray Diffraction Procedures for Crystalline and Amorphous Materials," Wiley, New York, 1954.

33. J. P. Pouget, M. E. Jozefowicz, A. J. Epstein, X. Tang, and A. G. MacDiarmid, Macromolecules, 24, 779 (1991).

34. Y. B. Moon, Y. Cao, P. Smith, and A. J. Heeger, Polym. Commun., 30, 196 (1991).

35. E. Emine, S. Mehmet, and K. Meral, Polym. Int., 39, 153 (1996). 
36. A. R. Hopkins, P. G. Rasmussen, and R. A. Basheer, Macromolecules, 29, 7838 (1996).

37. V. Prevost, A. Petit, and F. Pla, Synth. Met., 104, 79 (1999).

38. K. Suri, S. Annapoorni, R. P. Tandon, and N. C. Mehra, Synth. Met., 126, 137 (2002).
39. B. H. Sohn and R. E. Cohen, Chem. Mater., 9, 9264 (1997).

40. T. Nguyen and A. Diaz, Adv. Mater., 6, 858 (1994).

41. "JCPPS Powder Diffraction File International Center for Diffraction Data, $\mathrm{Fe}_{3} \mathrm{O}_{4}$," Newtown Square, PA, 1980. 